\title{
SUSCETIBILIDADE DE ALPHITOBIUS DIA PERIN US (PANZER, 1797) (COLEOPTERA: TENEBRIONIDAE) A REGULADORES DE CRESCIMENTO DE INSETOS (RCI)
}

\author{
A .M. Chernaki-Leffer'*, D .R. Sosa-G omez², L.M . de Almeida ${ }^{1}$ \\ 'Universidade Federal do Paraná, Departamento de Zoologia, Rua XV de Novembro 1299, CEP 80060-000, \\ Curitiba, PR, Brasil. E-mail: amcleffer@uol.com.br
}

RESUMO

\begin{abstract}
A toxicidadedosinseticidasreguladoresdecrescimentodeinsetos(IRC)dorfluazurom, triflumurom, diflubenzurom, lufenuromemetoxifenozidefoi avaliadaemcondiçõesdelaboratório paralarvasdo cascudinho, A lphitobius diaperinus (Panzer). A slarvas foram separadas em 2 grupos deacordo com o comprimento do corpo comolarvasmédias $(0,7 \mathrm{~cm})$ elarvasgrandes $(1,3 \mathrm{~cm})$, al imentadas com ração paracoel hostratadacomconcentraçõesentre1,6e200ppmdeingredienteativo(i.a).A $C_{50}$ paracada produto foi estimada por análisedeProbit. Osprodutosmaisativosforamclorfluazurom(valoresde $\mathrm{CL}_{50}$ atingiram de21.7 to $31.3 \mathrm{ppm} / \mathrm{g}$ dedieta) el ufenurom $\left(\mathrm{CL}_{50}\right.$ entre21,1 to $64,4 \mathrm{ppm} / \mathrm{g}$ dedieta). A CL $L_{50}$ para triflumurom (111,2 ppm/ g) e diflubenzurom (145,0 ppm/ g) foi 3,6 e 4,6 vezes, respectivamente, maisaltaqueparaclorfluazurom. Diflubenzuromemetoxifenozideapresentaram menor toxicidade. Metoxifenozidenasconcentrações de $160 \mathrm{ppm} /$ g dedietacausou mortalidadeentre 9,9 to $33,3 \%$. Na mesma concentração, a mortalidade por diflubenzurom foi de 39,9 to $53,3 \%$. Clorfluazurom, lufenurom etriflumurom podem ser promissores para futuros testes em aviários.
\end{abstract}

PALAVRAS-CHAVE: Cascudinho, triflumurom, clorfluazurom, metoxifenozide, toxicidade.

\section{ABSTRACT}

SUSCEPTIBILITY OF ALPHITOBIUS DIAPERINUS (PANZER) (COLEOPTERA: TENEBRIONIDAE) TO INSECT GROWTH REGULATOR (IGR). Toxicity to the insect growth regulators(IGR) chlorfluazuron, triflumuron, diflubenzuron, lufenuron and methoxyfenozidewas evaluated against larvae of the lesser mealworm, A Iphitobius diaperinus (Panzer) in laboratory conditions. Larvaewere separated into 2 groups according to their body length as middlelarvae $(0.7 \mathrm{~cm})$ and largelarvae $(1.3 \mathrm{~cm})$, fed with rabbit food treated with concentrations between 1.6 and 200 ppm to active ingredient (i.a). The LC $_{50}$ was estimate using Probit analysis. The most actives productswereclorfluazuron ( $\mathrm{LC}_{50}$ valuesranged from 21.7 to $31.3 \mathrm{ppm} / \mathrm{gdiet}$ ) and lufenuron ( $\mathrm{LC}_{50}$ values ranged from 21.1 to $64.4 \mathrm{ppm} / \mathrm{g}$ diet). The $L C_{50}$ for triflumuron $(111.2 \mathrm{ppm} / \mathrm{g})$ and diflubenzuron (145ppm/ g) were3.6and 4.6times, respectively, higher than that for chlorfluazuron. Diflubenzuron and methoxifenozi dewerelesstoxic. Methoxifenozidein theconcentrations of 160 $\mathrm{ppm} / \mathrm{g}$ of thediet caused amortal ity of between 9.9to 33.3\%. A tthesameconcentrationsmortalities by diflubenzuron wasfrom 39.9to53.3\%. Chlorfluazuron, lufenuron and triflumuron demonstrated promising results for futuretests in poultry houses.

KEY WORDS: Lesser mealworm, triflumuron, chlorfluazuron, methoxyfenozide, toxicity.

INTRODUÇÃO

Alphitobius diaperinus (Panzer), encontradoemaviáriosdefrangosdecorteemaltosníveispopulacionais, conhecido como "cascudinho" ou "besouro da cama", apresenta potencial vetorial na transmissão de patógenos aviários (VorIs et al., 1994; CHeRNAKI-LefFER et al., 2002), causando também prejuízos nas instalações dos gal pões de criação (ARENDS, 1987).
Sua presença em aviários tem motivado a ação de medidas de controle, como aplicação de inseticidas químicos nos gal pões de criação e método cultural, commanejo dacamaedosgal pões decriação (BELLANER et al., 2003).

Os produtos químicos utilizados com maior freqüênciasão os piretróides eorganofosforados, porém esteúltimo está em desuso, devido à possibilidade de deixar resíduosnacarcaça. São utilizadostambém, em

${ }^{2} E m p r a p a$ Centro Nacional de Pesquisa da Soja/ CNPSO, Londrina, PR, Brasil.

*Bolsista CN Pq/ Doutorado.

A rq. Inst. Biol., São Paulo, v.73, n.1, p.51-55, jan./ mar., 2006 
menor escala, osinibidoresdesíntesedequitina, denominadosinseticidasreguladoresdecrescimento (IRC).

Os IRC surgiram na década de 70 como um novo grupo deinseticidas deação mais específica emenor toxicidade para mamíferos do que os outros inseticidas (SILVA \& M ENDES, 2002), pois atuam seletivamente ao interromper o desenvolvimento eo crescimento ao invés de intoxicação direta.

As bezoilfeniluréias (diflubenzurom, lufenurom, teflubenzurom, entre outros) são os principais representantes dos IRC, interferindo, especificamente, na deposição dequitina, um dos compostos da cutícula de insetos (REYnolds, 1987). Exercem sua ação tóxica emformas imaturas (larvas), particularmentedurante a ecdise, impedindo o inseto de liberar-se da exocutícula, por não conseguirem secretar endocutícula nova (SiLva et al., 2003).

Um outro grupo de IRC, as diacilhidrazias (ex. metoxifenozideetebufenozide) atuam como agonistas deecdisteróides, provocando aceleração no processo da ecdise (Dhadialla, 1998).

A eficácia dosinibidores da formação decutícula sobrelarvas de A . diaperinus tem sido confirmada em condições de laboratório (EDWARDS \& ABRAHAM, 1985; Weaver \& Kondo, 1987; Miller \& Redfern , 1988; Demilo et al., 1995). Sua ação sobre os ovos desse coleóptero foi demonstrada por Mahmuda \& Parween (2000), ondea aplicação de do triflumurom sobre ovos deA. diaperinus produziu deformi dades noestágio adulto.

O emprego prático de IRC em aviários é pouco referido, exceto pelos estudos de WEAVER (1996) e SALIN et al. (2003), que relataram que o triflumurom apresenta resultados satisfatórios em experimentos de campo. De acordo com EdwARDs \& A BRAHAm (1985), IRC como o metopreneefenoxicarb, por exemplo, são debaixatoxicidadeavertebrados, podendo ser administrados via ração em criação defrangos, permanecendo biologicamente ativos mesmo após passagem no trato digestivo das aves.

No Brasil, algumas granjas avícolas fazem uso do triflumurom, entretanto, na literatura científica nacional nãotêmsido encontradosresultadossobre eficiência de controle.

Esta pesquisa teve como objetivo avaliar a suscetibilidadedelarvasdeA . diaperinusaosinseticidas clorfluazurom, lufenurom, triflumurom, diflubenzurom e metoxifenozide em condições de laboratório e comparar seu potencial inseticida.

\section{MATERIAL E MÉTODOS}

Coleta e manutenção dos insetos - A coleta de larvas de A. diaperinus foi realizada em aviários de frango de corte nos Municípios de Cascavel e Pato Branco, PR, Brasil. As slarvas foram mantidas em sala climatizada $\left(24 \pm 2^{\circ} \mathrm{C}\right.$, umidade relativa entre $60 \pm$ $10 \%$, escotofase $24 \mathrm{~h}$ ), em recipientes plásticos providos de aeração contendo maravalha e ração para coel hos levemente umedecidos com água destilada.

Bioensaios - Os compostos avaliados foram os reguladores de crescimento de insetos diflubenzurom, triflumurom, dorfluazurom, lufenuromemetoxifenozide O produto comercial foi diluído emágua destiladapara obtenção de diferentes concentrações do ingredienteativo em ppm, a saber: 1,6, 5, 10, 15, 25, 40, 45, 80, 120, 160e 200ppm. Decadaumadasconcentraçõesretirou-seuma alíquotade10mL quefoi adicionadaem50gderaçãopara coelhoseposteriormentetransferidos paracopos plásticos com capacidade para $500 \mathrm{~mL}$. Utilizaram-se duas categorias delanvas, separadasportamanho: larvascom aproximadamente 0,7 cm decomprimento (larvasmédias-Lm) elarvaspróximas àfasedepupa, comaproximadamente1,3cm(larvasgrandes-Lg). Foramtestadas105 larvas por repetição, com duas repetições para Lm e quatro repetições para Lg. A ração impregnada com inseticida foi substituída por ração umedecida em água destilada quando verificado o início de contaminação porfungos(apóstrêsdias). Emtodosostestesfoi utilizado um grupo testemunha, tratado somentecom água destilada. Os bioensaios foram conduzidos em câmaras dimatizadas ( $28^{\circ} \mathrm{C}, \mathrm{UR} \geq 70 \%$, escotofase $24 \mathrm{~h}$ ), durante 14dias, avaliando-sediariamenteonúmerodeindivíduos vivos emortos.

Análise Estatística - $A \mathrm{CL}_{50}$ foi cal culada utilizando-se o programa Polo-PC. A comparação entre os fatores inseticida-concentração e mortalidade entre larvasmédiasegrandesfoi feitaporanálisedevariância eteste $F(p<0,05)$, utilizando-seo Software $R$.

\section{RESULTADOSE DISCUSSÃO}

Em todos os testes foi observada mortalidade de larvas tratadas após $24 \mathrm{~h}$ deexposição, não havendo diferença significativa na porcentagem demortalidade entrelarvasmédiasegrandes. Observou-sediferença significativa na porcentagem de mortalidade de larvassubmetidas àsconcentraçõesde 10 e 160 ppm, não havendo, entretanto, interação significativa entre os fatores inseticida e concentração ao nível de significância de $5 \%$ (Tabela 1). Metoxifenozide causou mortalidademáximade $33,3 \%$ em larvasgrandes a 160 ppm, demonstrando ser pouco tóxico para larvas deA . diaperinus. Diflubenzurom causou mortalidade entre 39,9 e $53,3 \%$ na mesma dose, sendo que a 10 ppm a mortalidade não ultrapassou $13,3 \%$. Lufenurom causou mortalidade máxima de $56,7 \%$ a 10 ppm e63,3\% a 160 ppm. Triflumurom apresentou significativa diferençanaporcentagem demortalidade entre as doses de 10 e 160 ppm (10 e 76,7\%, respectivamente). Clorfluazurom causou mortalida- 
dede76,7\%a10ppm. A slarvasdogrupotestemunha apresentaram mortalidade de até $17,8 \%$ (Tabela 1).

As diferenças entre os inseticidas clorfluazurom, triflumurom elufenurom foram verificadas com base na mortalidade ao 8 o dia após adição do produto à ração. Para diflubenzurom e metoxifenozide, a heterogeneidade dos resultados não possibilitou a execução da análise, de forma que os dados não se ajustaram modelo de Probit.

A reduzida porcentagem de mortalidade obtida na concentração de 10 ppm para triflumurom e diflubenzurom contrastacomos resultados deMILLER $\&$ REDFERN (1988), que encontraram mortalidademaior que $90 \%$ em larvas de A . diaperinus que ingeriram 2 ppmdetriflumuromnaração equeodiflubenzurom causou mortalidademaior que $90 \%$ na concentração de5ppmna ração. No entanto, éimportantesal ientar no referido trabal ho, que as larvas ficaram durante todo período de avaliação (desde a eclosão até os 30 dias), noal imentotratado, oquepossi bilitouaingestão constante do ingrediente ativo. Nos bioensaios do presente estudo, as larvas ficaram expostas ao alimento tratado apenas durante 3 dias, sendo posteriormente al imentadas com ração não tratada.

As diferenças de toxicidade dos compostos para A . diaperinus foram avaliadas com base na $\mathrm{CL}_{50}$, que em larvas médias egrandes não foram significativamente diferentes para triflumurom, clorfluazurom e lufenurom, indicando que o tamanho das larvas não influenciou na toxicidade do produto no 8o dia de exposição ao químico. Clorfluazurom e lufenurom tiveram ação semel hante para larvas médias de $A$. diaperinus, (21,7 ppm e 21,1 ppm/g de ração, respectivamente). Contudo, triflumurom foi menos ativo, diferindo de clorfluazurom, porém estatisticamente semelhante ao lufenurom. Dentre os IRC testados para larvasgrandes, diflubenzuromapresentou $\mathrm{CL}_{50}$ de 145,0 ppm/ g, não diferindo estatisticamente de triflumurom elufenurom. Para clorfluazurom foi ob- tida $\mathrm{CL}_{50}$ de31,3ppm/ g, com resposta semel hanteao lufenurom $\left(\mathrm{CL}_{50}=64,4 \mathrm{ppm} / \mathrm{g}\right.$ ) (Tabela 2).

A baixa toxicidade do composto diflubenzurom paraA diaperinusjáhaviasido relatadaporGYGA(1987), queavaliou, em testesemaviários depostura, o efeitodo ciromazine e diflubenzurom para controle de M usca domestica (Linnaeus) (Diptera: Muscidae), ondeseobservou que populações de $A$. diaperinus que viviam em grande número no esterco não foram afetadas por nenhum dos químicos. Demaneira semelhante, MILLER \& R⿴囗十RN (1988) sugerem que este composto não seja adequado para controlarA . diaperinus, poisníveissignificativos dediflubenzuromsãoencontradosnostecidos eovos degal inhas poedeirasal imentadas com concentração de 10 ppm do inseticida na ração.

Tabela 1- M ortalidade (\%) delarvasmédias ( $\mathrm{LM}=0,7 \mathrm{~cm}$ ) egrandes ( $L G=1,3 \mathrm{~cm}$ decomprimento) deA. diaperinus no 70 dia após ingestão deração com diferentes produtos reguladoresdecrescimento deinsetos, nas concentrações de 10 e 160 ppm de i.a./ g de ração.

\begin{tabular}{lccc}
\hline IRC & $\begin{array}{c}\text { Ingredienteativo } \\
\text { (i.a.) em ppm/ g } \\
\text { de ração }\end{array}$ & \multicolumn{2}{c}{ Mortalidade(\%) } \\
\cline { 3 - 4 } & & LM & LG \\
& & $(\mathrm{n}=30)$ & $(\mathrm{n}=30)$ \\
\hline Triflumurom & 10 & 10,0 & 13,3 \\
Clorfluazurom & 160 & 76,7 & 46,7 \\
& 10 & 76,7 & 13,3 \\
Diflubenzurom & 160 & 59,9 & 63,3 \\
& 10 & 6,7 & 13,3 \\
Lufenurom & 160 & 39,9 & 53,3 \\
& 10 & 56,7 & 40,0 \\
Metoxifenozide & 160 & 63,3 & 43,3 \\
Testemunha & 10 & 13,3 & 20,0 \\
\hline
\end{tabular}

Tabela2-Suscetibilidadedelarvas médias (aproximadamente0,7cm) elarvas grandes (aproximadamente1,3cm) deA . diaperinus no 8ํำ dia após exposi ção a diferentes reguladores decrescimento de insetos.

\begin{tabular}{lccccc}
\hline Inseticidas & No. Larvas testadas & $\mathrm{CL}_{50}\left(\mathrm{IC}_{95 \%}\right) \mathrm{ppm} / \mathrm{g}$ ração & Coef. angular $\pm \mathrm{DP}$ & $\chi^{2}$ & g.I. \\
\hline Larvas médias & 180 & $73,3(42,2-101,1)$ & $2,1 \pm 0,5$ & $1,9^{\text {n.s. }}$ & 3 \\
Triflumurom & 150 & $21,7(7,0-37,9)$ & $1,2 \pm 0,3$ & $2,3^{\text {n.s. }}$ & 3 \\
Clorfluazurom & 285 & $21,1(6,1-44,8)$ & $0,6 \pm 0,2$ & $7,6^{\text {n.s. }}$ & 8 \\
Lufenurom & & & & & \\
Larvas grandes & 270 & $145,0(94,3-390,3)$ & $1,6 \pm 0,6$ & $0,2^{\text {n.s. }}$ & 4 \\
Diflubenzurom & 240 & $111,2(63,2-196,6)$ & $1,2 \pm 0,3$ & $2,4^{\text {n.s. }}$ & 4 \\
Triflumurom & 270 & $31,3(16,4-49,2)$ & $1,1 \pm 0,2$ & $2,2^{\text {n.s. }}$ & 4 \\
Clorfluazurom & 268 & $64,4(29,3-145,5)$ & $0,9 \pm 0,3$ & $2,2^{\text {n.s. }}$ & 4 \\
Lufenurom & & & & & \\
\hline
\end{tabular}

Heterogeneidade $=\chi^{2} /$ g.l.

$\chi^{2}(0,95)^{\text {n.s }}=$ não significativo. 
A maior toxicidadedoclorfluazuromquandocomparada ao triflumurom ediflubenzurom IRC foi também comprovada para Oryzaephilus surinamensis (Linnaeus) (Coleoptera: Silvanidae) (ELEK \& LonGSTAFF, 1994). Não hádadosnaliteraturasobreaeficiênciado clorfluazurom sobre A . diaperinus.

Demark \& Bennett (1989) compararam a eficiência dos compostos hexaflurom, triflumurom e clorfluazurom em ninfas de Blattella germanica (Linnaeus) (Dictyoptera: Blattellidae) e verificaram que a $\mathrm{CL}_{50}$ para hexaflurom e triflumurom contra ninfas dequinto ínstar foi maisque 1.000 vezes mais alta que para clorfluazurom. Embora com diferença menos expressiva, verificou-se que a $\mathrm{CL}_{50}$ do clorfluazuromfoi 3,4e3,5vezesmenor (larvasmédias e grandes, respectivamente), que para triflumurom, demonstrando, da mesma forma, a maior toxicidade do inseticida.

Observou-se que metoxifenozide apresentou baixa toxicidade para larvas médias e grandes de $A$. diaperinus (inferior a 50\%) em todos os intervalos de doses. Metoxifenozide é um produto do grupo das diacilhidrazinas e atuam como agonistas dos ecdisteróides, provocando acel eraçãono processo da ecdise (Омото, 2000). Os demais compostos testados, entretanto, são inibidores dasíntesedequitina. Desta forma, podemos inferir que os agonistas dos ecdisteróides parecem não apresentar atuação significante sobre as larvas deste coleóptero.

Conclui-se, portanto, que metoxifenozide e diflubenzurom não sejam bons candidatos para controle do cascudinho. Entretanto, clorfluazurom, lufenurom e também o triflumurom podem ser promissores para real ização de futuros testes para controle de A . diaperinus em aviários.

\section{AGRADECIMENTOS}

Ao Prof. MSc. A dilson dos A njos, (Departamento de Estatística, UFPR); aos técnicos da Embrapa Soja, JoséjairodaSilva, IvanildaSoldórioejovenil daSilva.

REFERÊNCIAS

ARendS, J.J. Control, management of the litter beetle. Poultry Digest, v.172, p.174-176, 1987.

Bellaver, C.; C osta, C.F.;F Igueiredo, E.A.P.; Aenisch, F.R.F.; Favero,J.A.;P alhares, J.C.P.;F IOREnTIN ,L.;Brum, P.A.R.; Abreu, P.G.; Avila, V.S. Boas práticas de produçao de frangos. Concórdia, 2003. (Circular Técnican.38).

Chernaki-Leffer, A.M.;Biesdorf,S.M.;A lmeida, L.M.; Leffer, E.V.B. \& VIGNE, F. Isolamento de enterobactérias em A I phitobius diaperinus ena cama deaviários no Oeste do Estado do Paraná, Brasil. R evista BrasileiradeCiência A vícola, v.4, n.3, p.243-247, 2002.
Demark, J.J. \& Bennett, G.W. Efficacy of chitin synthesis inhibitors on nymphal german cockroaches (Dictyoptera: Blattelidae). Journal of Economic Entomology, v.82, n.6, p.1633-1637, 1989.

Demilo,A.B.;M ILler, R.W.; Bordas, B.;Redfern ,R.E.; \& M ILls JUnIOR, G. Larvicidal effects of benzoylphenylureas against the lesser mealworm (Coleoptera: Tenebrionidae): quantitative structure-activity relationships. Journal of Entomological Science, v.30, n.3, p.324-332, 1995.

Dhadialla, T.S.; Carlson, G.R., Le, D.L. New insecticides with ecdysteroidal and juvenile hormone activity. A nnual Review of Entomology, v.43, p.545-569, 1998.

EDWARDS,J.P.\&A BRAHAM , L. Laboratory evaluation of two insectjuvenilehormoneanal oguesagai nstA I phitobius diaperinus (Panzer) (Coleoptera: Tenebrionidae). O urnal of Stored Product R esearch, v.21,n.4, p.189-194, 1985.

Elek, J.A. \& LongstafF, B.C. Effect of Chitin-Synthesis Inhibitors on stored-product beetles.PesticideScience, v.40, n.3, p.225-230, 1994.

GYGA, D.P. Evaluation of the insect growth regulators cyromazineand diflubenzuron assurfacespraysand feed additives for controlling housefliesM uscadomestica (L.) in chicken manure. International Pest Control, v.29, p.66-69, 1987.

Mahmuda, B. \& ParWEen, S. Latent effect of egg treatment with triflumuron on morphogenesis in A lphitobius diaperinus Panzer (Coleoptera: Tenebrionidae). Bangladesh Journal of Entomology, v.10, n.1/2, p.121-124, 2000.

MILLER, R.W. \& REDFERN , R.E. Feed additives for control of lesser mealworm (Coleoptera: Tenebrionidae) in poultry broiler houses. J ournal of E conomic Entomology, v.81, n.4, p.1137-1139, 1988.

Омото, C. Modo de ação de inseticidas e resistência de insetos a inseticidas. In: Guedes, C.; Costa, I.D.; Castiglioni, E. (Eds.). Bases e técnicas do manejo de insetos. Santa Maria: Pall otti, 2000. 248p.

REYNOLDS, S.E. Thecuticule, growthregulatorsand mouting in insects: the essential background to the action of acylurea insecticides. P esticideS cience, v.20, p.131-146, 1987

Salin, C.; Delettre, Y.R.; Vernon, P. Controlling the mealworm Alphitobius diaperinus (Coleoptera: Tenebrionidae) in broiler and turkey houses: field trials with a combined insecticide treatment: Insect Growth Regulator and Pyrethroid.J ournal of E conomic Entomology, v.96, n.1, p.126-130, 2003.

SiLVA, J.J. \& MENDES, J. Effect of diflubenzuron on stages of Hematobia irritans (L.) (Diptera, Muscidae) in Uberlândia, Stateof Minas Gerais, Brasil.M emóriasdo Instituto 0 swaldo Cruz, v.97, p.679-682, 2002.

Silva, M.T.B.; Costa, E.C.; Boss, A. Control of Anticarsia gemmatalis Hübner (Lepidoptera: Noctuidae) larvae with insect growth regulators. Ciência Rural, v.33, n.4, p.601-605, 2003.

VorIS, J.C.;M EYER, J.A.; PFost,R.;W oOdBURY, R. Temperature affects lesser mealworm populations in turkey brooder houses. CaliforniaA griculture, v.48, n.2, p.1821, 1994. 
WEAVER,J.E.\&K ONDO, V.A. Laboratory evaluation of insect growth regulators in producing lesser mealworm mortality and egg infertility. Journal of A gricultural Entomology, v.4, n.3, p.233-245, 1987.

WEAVER, J.E. The lesser meal worm, Al phitobius diaperinus: Field trails for control in a broiler house with insect growth regulators and pyrethroids. Journal of A gricultural Entomology, v.13, n.2, p.93-97, 1996.

Recebido em 26/ 12/ 05

Aceito em 10/ 2/ 06 